\title{
Numerical Study of Novel Ratiometric Sensors Based on Plasmon-Exciton Coupling
}

\author{
Yuankai Tang', Xiantong Yu', Haifeng Pan', Jinquan Chen', \\ Benjamin Audit ${ }^{2}$, Françoise Argoul ${ }^{4}$, Sanjun Zhang ${ }^{1,3}$, and \\ Jianhua $\mathbf{X} \mathbf{u}^{\prime}$
}

\begin{abstract}
We numerically studied the optical properties of spherical nanostructures made of an emitter core coated by a silver shell through the generalized Mie theory. When there is a strong coupling between the localized surface plasmon in the metallic shell and the emitter exciton in the core, the extinction spectra exhibit two peaks. Upon adsorption of analytes on these core-shell nanostructures, the intensities of the two peaks change with opposite trends. This property makes them potential sensitive ratiometric sensors. Molecule adsorption on these nanostructures can be quantified through a very simple optical configuration likely resulting in a much faster acquisition time compared with systems based on the traditional metal nanoparticle surface plasmon resonance (SPR) biosensors.
\end{abstract}

\section{Keywords}

Surface plasmon resonance, nanoparticles, biosensor, strong coupling

\section{Introduction}

Localized surface plasmon resonance (LSPR) is the resonance of oscillating excitation light with localized surface plasmon (LSP) in confined metal nanostructures. It has attracted the attention of many researchers due to its wide range of applications including surface-enhanced Raman scattering, ' plasmon-enhanced fluorescence, ${ }^{2}$ and modified spontaneous emission. ${ }^{3}$ Interactions between LSP and the surrounding environment can significantly change the resonance frequency. This makes, for example, LSPR sensitive to the change of local refractive index ${ }^{4}$ and to biomolecules adsorption on nanostructure surface. ${ }^{1,5}$ Various LSPRbased sensors have been designed based on gold and silver nanostructures of diverse shapes like star-shaped gold nanoparticles, ${ }^{6}$ silver nanocubes, ${ }^{7}$ gold nanorods, ${ }^{5}$ gold-silica-gold nanoshells, ${ }^{8}$ silver nanoparticles, ${ }^{4}$ goldair-gold nanoparticles, and silver-air-silver nanoparticles. ${ }^{9}$ Most LSPR-based sensory systems operate via the measurement of a peak frequency shift in the extinction or scattering spectra. ${ }^{10}$

In this paper, we introduce a novel ratiometric core-shell sensor based on strong coupling between shell LSP and emitter exciton, allowing to quantify a local refractive change in the surrounding of the sensor through the intensity ratio of two extinction peaks. We used Mie's theory which has been demonstrated to be an effective method to model the optical properties of spherical core-shell nanoparticles ${ }^{1 \mathrm{I}-13}$ and the strong coupling between plasmons and excitons. ${ }^{14-16}$ We numerically studied the scattering, absorption, and extinction spectra of an emitter core with a silver shell (emitter@silver core-shell) nanostructure. When there is a strong coupling between the shell LSP and the emitter exciton, the spectra exhibit two peaks resulting from the plasmon polariton splitting.

\footnotetext{
'State Key Laboratory of Precision Spectroscopy, East China Normal University, Shanghai, China

${ }^{2}$ Univ Lyon, Ens de Lyon, Université Claude Bernard Lyon I, CNRS, Laboratoire de Physique, Lyon, France

${ }^{3}$ NYU-ECNU Institute of Physics at NYU Shanghai, 3663 Zhongshan Road North, Shanghai, China

${ }^{4}$ LOMA, Université de Bordeaux, CNRS, UMR 5798, Talence, France
}

Corresponding authors:

Sanjun Zhang, State Key Laboratory of Precision Spectroscopy, East China Normal University, Shanghai, 200062, China.

Email: sjzhang@phy.ecnu.edu.cn

Benjamin Audit, Univ Lyon, Ens de Lyon, Université Claude Bernard Lyon I, CNRS, Laboratoire de Physique, F-69342 Lyon, France.

Email: benjamin.audit@ens-lyon.fr 
The intensities of the two peaks change with opposite trends upon adsorption of analytes on the core-shell surface. This makes emitter@silver core-shell nanostructures potential sensitive ratiometric sensors. We discuss a very simple optical measurement configuration to quantify molecule adsorption on the sensor surface, likely presenting a much faster acquisition time compared with configurations based on the traditional metal nanoparticle sensors.

\section{Method of Numerical Simulation}

Mie's theory has been widely used to describe light scattering from homogeneous spherical particles. It was generalized to spheres made of an arbitrary number of homogeneous layers: first in 1985 by Bhandari that developed a matrix formalism to compute the scattering coefficients for a multilayered spheres ${ }^{17}$ and more recently by Sinzig and Quinten that presented a recursive formalism to compute these scattering coefficients. ${ }^{18}$ We employed the latter formalism that is more practical for numerical calculations.

As in Sinzig et al., ${ }^{18}$ we considered core-shell structures with $r$ homogeneous layers (Fig. I) of refractive indices $n_{s}$ $(s=\mathrm{I}, \ldots, r)$, embedded in a non-absorbing medium with real refractive index $n_{r+1}$. When they are illuminated by an unpolarized incident plane wave of vacuum wavelength $\lambda$, so that in each medium the corresponding wavenumber is $k_{s}=2 \pi n_{s} / \lambda$, the cross-sections of scattering $C_{s c a}$, extinction $C_{\text {ext }}$, and absorption $C_{a b s}$ can be written as: ${ }^{18}$

$$
\begin{aligned}
& C_{s c a}=\frac{2 \pi}{k_{r+1}^{2}} \sum_{n=1}^{\infty}(2 n+1)\left(\left|a_{n}\right|^{2}+\left|b_{n}\right|^{2}\right) \\
& C_{\text {ext }}=\frac{2 \pi}{k_{r+1}^{2}} \sum_{n=1}^{\infty}(2 n+1) \operatorname{Re}\left(a_{n}+b_{n}\right) \\
& C_{a b s}=C_{\text {ext }}-C_{\text {sca }}
\end{aligned}
$$

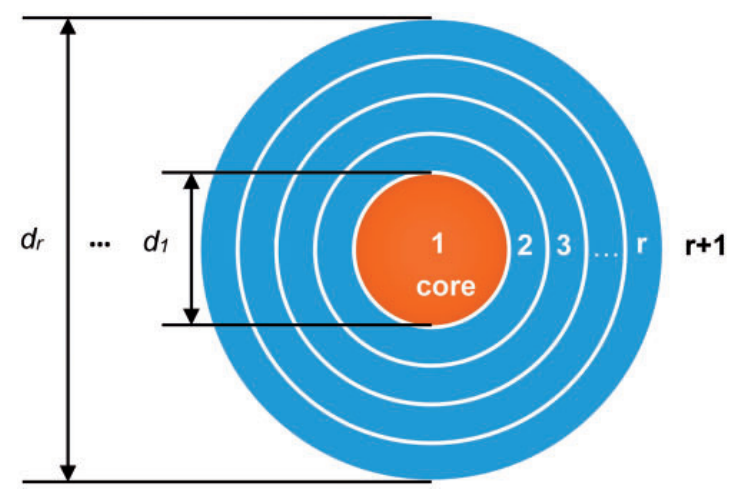

Figure I. Scheme of the core shell structure with $r$ homoge neous layers. Innermost layer (s I) is the spherical core, layers $s \quad 2, \ldots, r$ are $r-I$ shells and layer $s \quad r+I$ is the external medium. $d_{s}$ is the external diameter of the sth layer $(s \quad, \ldots, r)$. where $a_{n}$ and $b_{n}$ are related to the decomposition of the scattered electromagnetic field on the spherical vector harmonics of order $n$. Writing $y_{s}=k_{s} d_{s}$ where $d_{s}$ is the external diameter of the sth layer and $m_{s}=n_{s+1} / n_{s}$ for $s=1$. .r, the coefficients $a_{n}$ and $b_{n}$ can be computed as: ${ }^{18}$

$$
\begin{gathered}
m_{r} \psi_{n}\left(m_{r} y_{r}\right)\left[\psi_{n}^{\prime}\left(y_{r}\right)+T_{n}^{r}{ }^{1} \chi_{n}^{\prime}\left(y_{r}\right)\right] \\
a_{n}=-\frac{-\psi_{n}^{\prime}\left(m_{r} y_{r}\right)\left[\psi_{n}\left(y_{r}\right)+T_{n}^{r}{ }^{1} \chi_{n}\left(y_{r}\right)\right]}{m_{r} \xi_{n}\left(m_{r} y_{r}\right)\left[\psi_{n}^{\prime}\left(y_{r}\right)+T_{n}^{r}{ }^{1} \chi_{n}^{\prime}\left(y_{r}\right)\right]} \\
-\xi_{n}^{\prime}\left(m_{r} y_{r}\right)\left[\psi_{n}\left(y_{r}\right)+T_{n}^{r}{ }^{1} \chi_{n}\left(y_{r}\right)\right]
\end{gathered}
$$

and

$$
\begin{gathered}
\psi_{n}\left(m_{r} y_{r}\right)\left[\psi^{\prime}{ }_{n}\left(y_{r}\right)+S_{n}^{r}{ }^{1} \chi_{n}^{\prime}\left(y_{r}\right)\right] \\
b_{n}=-\frac{-m_{r} \psi_{n}^{\prime}{ }_{n}\left(m_{r} y_{r}\right)\left[\psi_{n}\left(y_{r}\right)+S_{n}^{r}{ }^{1} \chi_{n}\left(y_{r}\right)\right]}{\xi_{n}\left(m_{r} y_{r}\right)\left[\psi^{\prime}{ }_{n}\left(y_{r}\right)+S_{n}^{r}{ }^{1} \chi_{n}^{\prime}\left(y_{r}\right)\right]} \\
-m_{r} \xi_{n}^{\prime}\left(m_{r} y_{r}\right)\left[\psi_{n}\left(y_{r}\right)+S_{n}^{r}{ }^{1} \chi_{n}\left(y_{r}\right)\right]
\end{gathered}
$$

where $r-1$ is the number of shells, $\psi_{n}, \chi_{n}$ and $\xi_{n}$ are the Riccati-Bessel functions: $\psi_{n}(z)=z j_{n}(z), \chi_{n}=z y_{n}(z)$, $\xi_{n}=z h_{n}^{(1)}(z)$, where $j_{n}$ and $y_{n}$ are the first and second kind spherical Bessel functions, respectively, and $h_{n}^{(1)}$ the first kind spherical Hankel function and the prime denotes derivation of the function. Finally, coefficients $T_{n}^{r}{ }^{1}$ and $S_{n}^{r}{ }^{1}$ can be computed recursively using the following expressions: ${ }^{18}$

$$
\begin{gathered}
T_{n}^{1}=-\frac{m_{1} \psi_{n}\left(m_{1} y_{1}\right) \psi_{n}^{\prime}\left(y_{1}\right)-\psi_{n}^{\prime}\left(m_{1} y_{1}\right) \psi_{n}\left(y_{1}\right)}{m_{1} \chi_{n}\left(m_{1} y_{1}\right) \psi_{n}^{\prime}\left(y_{1}\right)-\chi_{n}^{\prime}\left(m_{1} y_{1}\right) \psi_{n}\left(y_{1}\right)} \\
T_{n}^{s}=-\frac{-\psi_{n}^{\prime}\left(m_{s} y_{s}\right)\left[\psi_{n}\left(y_{s}\right)+T_{n}^{s}{ }^{1} \chi_{n}\left(y_{s}\right)\right]}{m_{s} \chi_{n}\left(m_{s} y_{s}\right)\left[\psi^{\prime}{ }_{n}\left(y_{s}\right)+T_{n}^{s}{ }^{1} \chi^{\prime}{ }_{n}\left(y_{s}\right)\right]} \\
-\psi^{\prime}{ }_{n}^{\prime}\left(y_{s}\left(m_{s} y_{s}\right)\left[\psi_{n}\left(y_{s}\right)+T_{n}^{s}{ }^{1} \chi_{n}\left(y_{s}\right)\right]\right. \\
S_{n}^{1}=-\frac{\psi_{n}\left(m_{1} y_{1}\right) \psi^{\prime}{ }_{n}\left(y_{1}\right)-m_{1} \psi^{\prime}{ }_{n}\left(m_{1} y_{1}\right) \psi_{n}\left(y_{1}\right)}{\chi_{n}\left(m_{1} y_{1}\right) \psi^{\prime}{ }_{n}\left(y_{1}\right)-m_{1} \chi^{\prime}{ }_{n}\left(m_{1} y_{1}\right) \psi_{n}\left(y_{1}\right)} \\
S_{n}^{s}=-\frac{\psi_{n}\left(m_{s} y_{s}\right)\left[\psi^{\prime}{ }_{n}\left(y_{s}\right)+S_{n}^{s}{ }^{1} \chi_{n}^{\prime}\left(y_{s}\right)\right]}{\chi_{n}\left(m_{s} y_{s}\right)\left[\psi_{n}^{\prime}{ }_{n}\left(y_{s}\right)+S_{n}^{s}{ }^{1} \chi_{n}^{\prime}\left(y_{s}\right)\right]} \\
-m_{s} \chi_{n}^{\prime}\left(m_{s} y_{s}\right)\left[\psi_{n}\left(y_{s}\right)+S_{n}^{s}{ }^{1} \chi_{n}\left(y_{s}\right)\right]
\end{gathered}
$$

We used Eqs. I-7 to numerically estimate the cross-section versus wavelength spectra of emitter@silver core-shell nanostructures in order to study the behavior of the LSPR of the silver shell in the diverse geometries of interest. In Eqs. $\mathrm{I}-3$, the cross-sections are the sum of infinite number of terms. Hence, a suitable summation cutoff has to be 

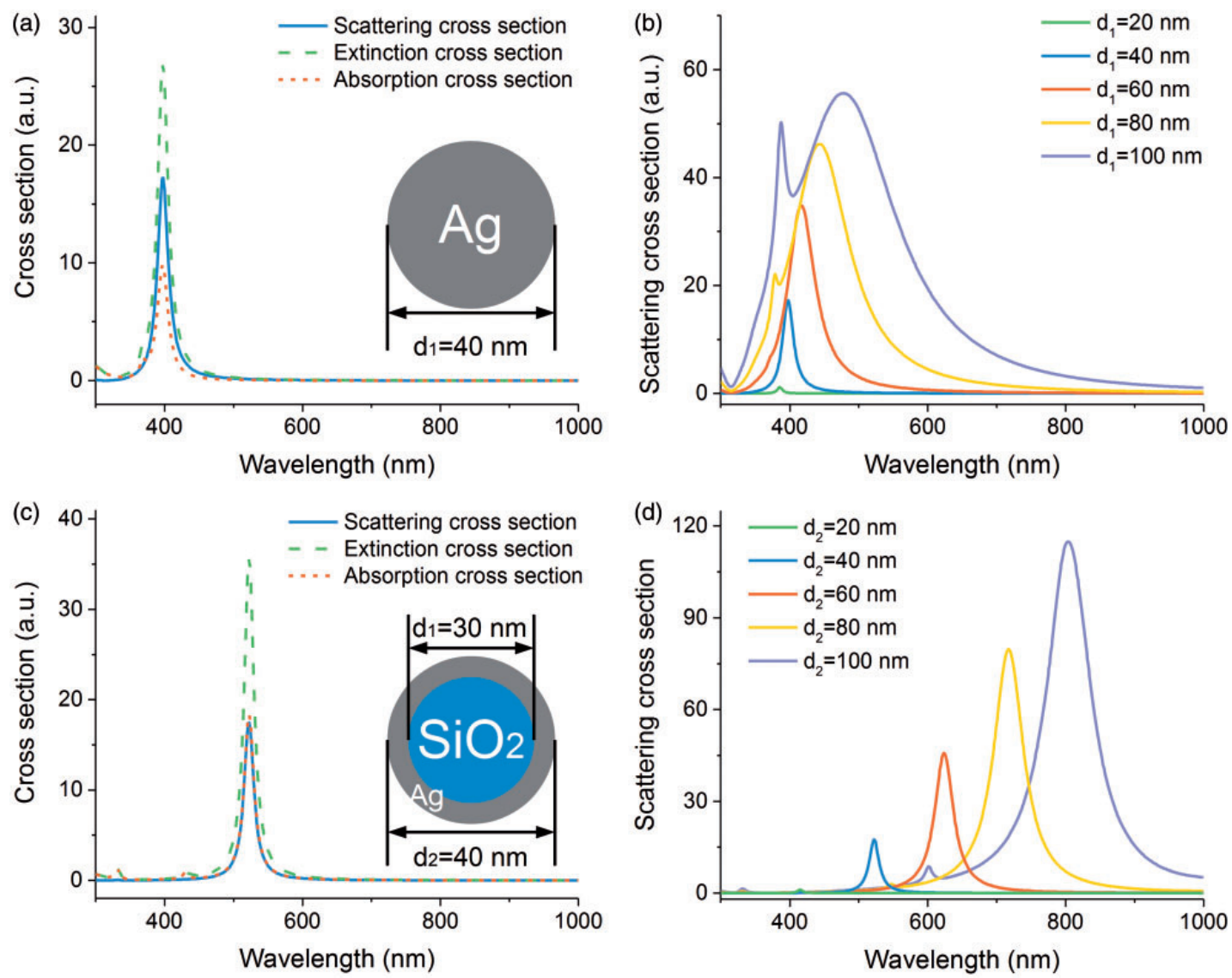

Figure 2. (a) Scattering, extinction, and absorption spectra of a silver sphere in water; the diameter is $d_{1} \quad 40 \mathrm{~nm}$. (b) Scattering cross section spectra of silver sphere of different size. (c) Scattering, extinction, and absorption spectra of a SiO ${ }_{2} @ A g$ core shell nanos tructure in water; the diameters are $d_{1} 30 \mathrm{~nm}$ and $d_{2} \quad 40 \mathrm{~nm}$. (d) Scattering cross section spectra of $\mathrm{SiO}_{2} @ \mathrm{Ag}$ core shell nanos tructures of different sizes, the thickness of the silver shell was kept at $5 \mathrm{~nm}$.

chosen that ensure calculation convergence. Terms of index $n$ correspond to the $n$th order eigenmode: dipole $n=1$, quadrupole $n=2$, octupole $n=3$, and so on. ${ }^{19}$ The general trend is that higher order eigenmodes become more important with increasing nanoparticle size. For example, when the radius of the $\mathrm{Ag}$ sphere is less than $20 \mathrm{~nm}$, the quadrupole has negligible contribution to the extinction cross section of the $\mathrm{Ag}$ spheres. However, when the radius is larger than $20 \mathrm{~nm}$, the quadrupole turns on in the extinction spectrum, and when the radius is larger than $60 \mathrm{~nm}$, the octopole has also to be considered. We performed these numerical calculations using Matlab and set $n=10$ to avoid missing any high order eigenmodes.

Generalized Mie's theory is thus an operational way to compute optical cross-sections of nanoparticle with core-shell structure, ${ }^{8,9, \mid 1-13,20,21}$ capturing special phenomena related to LSPR, such as strong coupling ${ }^{14,15,22}$ and Spasers. ${ }^{23,24}$ The cross-section calculations using our implementation of Sinzig and Quinten formalism agreed well with these reports. These observations prompted us to use Sinzig and Quinten formalism to investigate the strong coupling between LSPR and exciton in core-shell structure in this research.

\section{Results and Discussion}

\section{Optical Properties of $\mathrm{SiO}_{2} @ \mathrm{Ag}$ Core-Shell Nanoparticles}

We began by studying the LSPR properties of silver nanoparticles by computing their optical cross-section spectra as a function of wavelength using our implementation of the generalized Mie theory. The refractive index of silver was obtained from McPeak et al. ${ }^{25}$ Figure 2 a shows the calculated optical cross-sections of a spherical Ag nanoparticle of diameter $40 \mathrm{~nm}$. The optical spectra present a resonance wavelength at $395 \mathrm{~nm}$, which is in good agreement with the extinction peak of $\mathrm{Ag}$ nanoparticles in water $(\sim 400 \mathrm{~nm}){ }^{26}$ Note that the sensitivity of this resonance wavelength to the surrounding environment has been widely used to utilize $\mathrm{Ag}$ nanoparticles as label-free biosensors. ${ }^{26,27}$ With increasing size of the silver particle, the resonance peak in cross-section spectra is red shifted as demonstrated in Fig. $2 \mathrm{~b}$. When the diameter is larger than $80 \mathrm{~nm}$, a quadrupole resonance peak becomes apparent at a shorter wavelength $(\sim 390 \mathrm{~nm})$, in accordance with experimental results. $^{28}$ 
The refractive index of $\mathrm{SiO}_{2}$ has been measured as 1.46 in the wavelength range of $300-1000 \mathrm{~nm},{ }^{29}$ allowing us to numerically compute the cross-section spectra of $\mathrm{SiO}_{2} @ \mathrm{Ag}$ core-shell nanostructures (Fig. 2c, d). The $\mathrm{SiO}_{2}$ core significantly changes the optical properties compared with pure Ag nanoparticles of same size (Fig. 2). The resonance peak is red-shifted and becomes narrower, and the quadrupole resonance is suppressed. The resonance wavelength of the $40 \mathrm{~nm}$-diameter $\mathrm{SiO}_{2} @ \mathrm{Ag}$ nanostructure (Fig. 2c) is longer than that of the $100 \mathrm{~nm}$-diameter $\mathrm{Ag}$ nanoparticles, but the width of the resonance peak is tenfold narrower. This signifies that $\mathrm{SiO}_{2} @ \mathrm{Ag}$ core-shell structures have a longer decay time of LSPR compared to pure silver particles with the same resonance wavelength. ${ }^{30}$

J-aggregated cyanine dye, 5,5',6,6'-tetrachloroI, I'-diethyl-3,3'-di(4-sulfobutyl)-benzimidazolocarbocyanine (TDBC), is commonly used in strong coupling experiments between emitter and surface plasmon polariton (SPP) ${ }^{31,32}$ or LSPR ${ }^{33-37}$ because of its narrow absorption band and large oscillator strength. ${ }^{38}$ Strong coupling between LSPR and emitter excitons requires both that the frequency of LSPR to be close to the absorption bands of excitons and the emitter has a narrow absorption peak. The crosssection spectra discussed above show that tuning the LSPR resonance wavelength of a $\mathrm{Ag}$ sphere to $590 \mathrm{~nm}$, the absorption wavelength of TDBC, the resonance peak becomes so wide that strong coupling cannot be achieved (Figs. 2a, b). However, using silver as the shell of a core-shell nanoparticle appears as a suitable solution to this problem that can keep a sufficiently narrow resonance peak and allow the tuning of the resonance wavelength over hundreds of nanometers (Figs. 2c, d). ${ }^{39}$ Thus, core-shell nanostructure is a good candidate to obtain strong coupling between plasmons and excitons.

\section{Strong Coupling Between TDBC Core and Silver Shell}

The strong interaction between LSPR and emitter leads to plasmon-exciton states behaving like vacuum Rabi splitting, i.e., splitting of energy levels and change of peak positions. ${ }^{14,33,35-37,40-42}$ There are three models to describe the strong coupling between plasmon and exciton, namely, classical, semi-classical, and quantum description. Mie's theory describes light as a classical field and uses the refractive index to account for the material properties based on Maxwell's theory. Here, we used TDBC as the active material to form exciton. A Lorentz model is used to describe the permittivity of TDBC: ${ }^{31}$

$$
\varepsilon_{T D B C}(e)=\varepsilon_{m}(e)+\frac{f q^{2} \hbar^{2}}{m \varepsilon_{0} L_{z}} \frac{1}{e_{0}^{2}-e^{2}-i \gamma_{0} e}
$$

where $\hbar$ is the reduced Planck constant, $\varepsilon_{m}$ is the dielectric constant of the matrix in which the TDBC is doped, $e$ is the energy, $e_{0}=2100 \mathrm{meV}$ is the absorption energy of TDBC, $f=1.8 \times 10^{18} \mathrm{~m}^{-2}$ is the oscillator strength of the J-aggregate, $\gamma_{0}=49 \mathrm{meV}$ is the the exciton linewidth, $q$ the electron charge magnitude, $m$ the electron mass, $\varepsilon_{0}$ the permittivity of free space, and $L_{z}$ the thickness of the TDBC. The imaginary part of the permittivity shows that absorption wavelength of TDBC is $590 \mathrm{~nm}$.

We numerically computed the absorption, extinction, and scattering cross-section spectra of TDBC@Ag coreshell nanostructures in water while keeping the thickness of the silver shell at $5 \mathrm{~nm}$ (Fig. 3). For the particle with $d_{2}=50 \mathrm{~nm}$, a clear Rabi splitting of $110 \mathrm{meV}(\sim 30 \mathrm{~nm})$ is observed, characteristic of strong coupling between plasmon and exciton in a TDBC@Ag core-shell nanostructure in water (Fig. 3a). Changing the diameter of the TDBC core while keeping the thickness of the $\mathrm{Ag}$ shell constant allow us to tune the frequency of LSPR to different wavelengths. Increasing the particle size, the two split peaks redshift and their intensities change with opposite trends due to the interaction between LSPR and emitter exciton. The central wavelengths $\omega \pm$ of the two split peaks depend on the resonance wavelength $\omega_{p l}$ of LSPR and the absorption wavelength $\omega_{0}$ of $\mathrm{TDBC}^{33}$ as:

$$
\omega_{ \pm}=\frac{1}{2}\left(\omega_{p l}+\omega_{0}\right) \pm \sqrt{g^{2}+\delta^{4} / 4}
$$

where $g$ is the coupling rate and $\delta$ is the detuning, $\delta=\omega_{p l}-\omega_{0}$. In fact, Eq. 9 is a description of Rabi splitting. The anti-crossing of the $\omega_{ \pm}$versus diameter curves observed in the inserts of Figs. 3b-d can been seen in strong coupling or weak coupling regime (e.g., induced transparency). ${ }^{14}$ To discriminate which regime the plasmon-exciton interaction locates, we extract the coupling rate $g$ by equation: ${ }^{4}$

$$
\left(\omega_{+}-\omega\right)^{2}=\left[\begin{array}{c}
\left(\omega_{p l}-\omega_{0}\right)^{2}\left(\Gamma_{p l}-\gamma_{0}\right)^{2} \\
+4 g^{2}+\left(\omega_{p l}-\omega_{0}\right)^{2}-\frac{\left(\Gamma_{p l}-\gamma_{0}\right)^{2}}{4}
\end{array}\right)^{2}
$$

where $\Gamma_{p l}$ is the full width half-maximum (FWHM) linewidths of the plasmon. The coupling rate $g$ in Fig. 3 changes from $63 \mathrm{meV}$ to $49 \mathrm{meV}$ oppositely with the size of nanoparticle (Fig. SI), which reaches strong coupling regime according to $g^{2}>\left(\Gamma_{p l}^{2}+\gamma_{0}^{2}\right) / 16$ (Table SI). ${ }^{4 \mathrm{I}}$ It indicates that peak splitting is caused by plasmon-exciton strong coupling. However, increase of exciton material $\gamma_{0}$ and decrease of oscillator strength $f$ decrease coupling rate $g$. In such circumstance, the peak splitting becomes unobvious, and consequently not suitable for ratiometric measurement (Fig. S2). This numerical analysis shows that, by adapting the diameter of the exciton core and the thickness of the metal shell, the resonance frequency of LSP can easily 

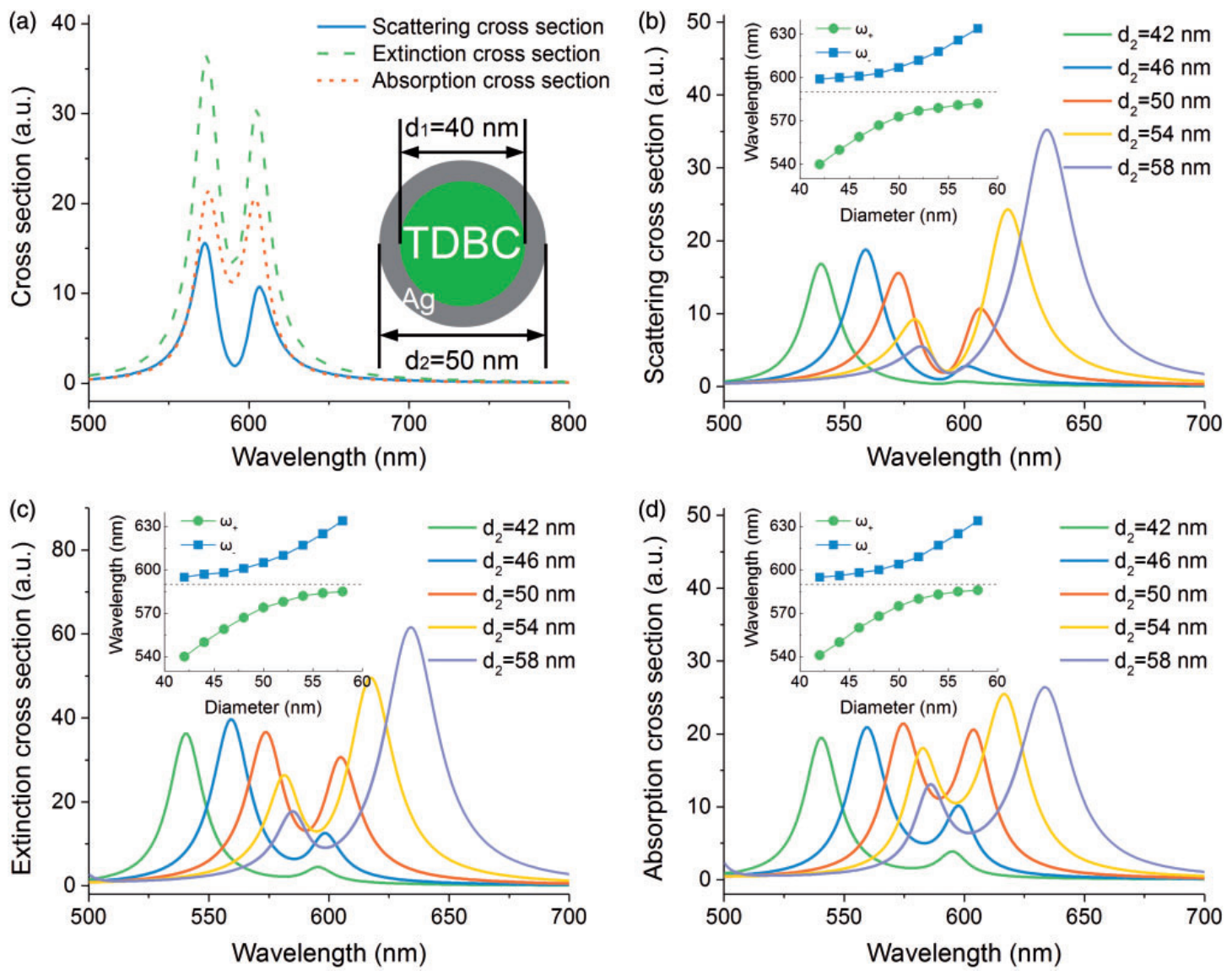

Figure 3. (a) Scattering, extinction and absorption cross section spectra of a TDBC( $\left.d_{1} \quad 40 \mathrm{~nm}\right) @ \mathrm{Ag}\left(d_{2} \quad 50 \mathrm{~nm}\right)$ core shell nano particle in water. (b-d) Spectra of scattering cross section (b), extinction cross section (c), and absorption cross section (d) of TDBC@Ag nanostructures in water with different core size. The thickness of the Ag shell was kept at $5 \mathrm{~nm}$. Inserts in (b-d) are the position of the two split peaks as a function of nanoparticle diameter.

be tuned to match the absorption frequency of the exciton. In our case of TDBC@Ag core-shell nanostructures, the shape of the two peaks is similar and symmetric around the central wavelength $\sim 590 \mathrm{~nm}$ when the diameter of TDBC core and the thickness of $\mathrm{Ag}$ shell are $4 \mathrm{Inm}$ and $5 \mathrm{~nm}$, respectively. In practice, this kind of core-shell nanostructures can easily be prepared by reducing silver nitrate on the emitter doped $\mathrm{SiO}_{2}$ nanoparticles. ${ }^{43-46}$

\section{Using TDBC@Ag Core-Shell Nanostructures to Detect the Change of Local Refractive Index}

The above discussion shows that the strong coupling in core-shell nanostructures is sensitive to LSPR. This property can be used in sensing application because the LSPR is influenced by the particle environment. When molecules adsorb on the core-shell nanostructure, the local refractive index near the nanoparticle is changed. This can be taken into account in our numerical formalism by considering an extra shell of refractive index $n_{s}$, whose thickness was fixed to $2 \mathrm{~nm}$ (Fig. 4a). When the refractive index of the surrounding shell changes, the extinction spectrum of the strong coupling core-shell nanostructure changes as well. Figs. $4 \mathrm{~b}$ and $\mathrm{c}$ shows the variation of the intensities and the wavelengths of the two split peaks as a function of the surrounding shell refractive index $n_{s}$. With the increase of $n_{s}$, the intensities of the two peaks change with opposite trends. Meanwhile, the wavelength of both peaks redshifts without crossing the 590-nm line, which is the absorption wavelength of TDBC. This feature can be used to perform ratiometric measurement, and the optical configuration is schematically illustrated in Fig. S3. Figure 4d shows the computed ratio of intensities of the two peaks versus the refractive index of surrounding shell $\left(n_{s}\right)$. When analytes adsorb on the surface, the local index changes, so does the ratio. The ratio changes with respect to the thickness of analyte shell (Figs. S4a and S4b). The thicker the analyte shell is, the larger the ratio changes. Furthermore, when the analyte shell is fixed to $2 \mathrm{~nm}$, changing the thickness of the silver shell or the diameter of the TDBC core can tune the spectra (Figs. S4c and S4e), the intensity ratio still 

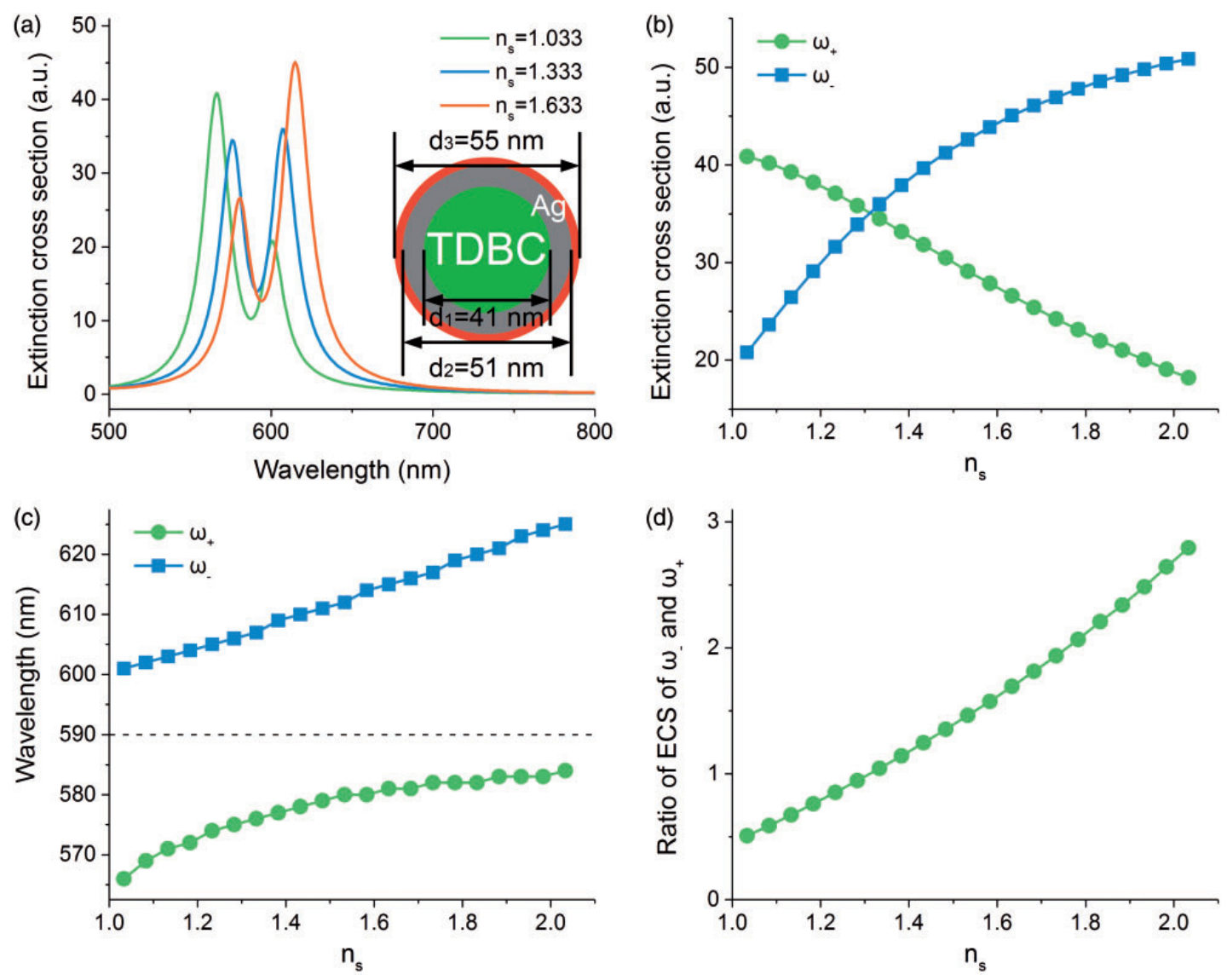

Figure 4. TDBC@Ag nanoparticle $\left(d_{1} \quad 4 \mathrm{lnm}, d_{2} \quad 5 \mathrm{Inm}\right)$ coated by a $2 \mathrm{~nm}$ thick shell of adsorbed material of refractive index $n_{s}$. (a) Extinction cross section spectra in water for different local refractive index $n_{s}$. (b) Intensity of the split peaks as a function of $n_{s}$. (c) Wavelength of split peaks as a function of $n_{s .}$ (d) Ratio of intensity $I_{+} / I_{-}$of the two split peaks as a function of $n_{s}$.

varies as a function of $n_{s}$ (Figs. S4d and S4f). Since the detection employs the ratio of transmitted and reflected intensities, the intensity fluctuation of incident white light is eliminated. This is a big advantage over traditional LSPR sensors. For comparison, we simulated the response of traditional LSPR Ag sensor of $5 \mathrm{I} \mathrm{nm}$ in the presence of a shell of adsorbed molecules (Fig. S5). When the refractive index of the surrounding shell $\left(n_{s}\right)$ increases, the spectrum intensity changes and the peak shifts to a longer wavelength. However, the intensity variation cannot be used in practice to quantify the change in the surrounding shell because it is influenced by many factors, such as, the concentration of nanoparticles and the fluctuation of light source. Hence, the variation in the surrounding shell is usually quantified by the peak wavelength of the extinction spectrum. This implies that light has to be dispersed in a spectrometer in order to acquire the full spectrum, which is time-consuming. In contrast, the measurement framework (Fig. S3) that can be deployed when using core-shell nanostructures is simpler, and the acquisition time is much shorter because the response of many commercial photodetectors can exceed $\mathrm{GHz}$, which is orders of magnitude higher than those used in full spectrum, such as charge-coupled device (CCD).
Hence, in principle, core-shell nanostructures presenting strong coupling between the shell LSP and the core emitter plasmon have unparalleled advantages over traditional LSPR sensors in the characterization of fast dynamic processes.

\section{Conclusion}

We numerically studied the strong coupling between LSPR and exciton in emitter@Ag core-shell nanostructures through generalized Mie theory. In this geometry, the resonance frequency of LSP in the Ag shell can easily be tuned to match the absorption frequency of the core exciton by changing the diameter of the emitter core and the thickness of the Ag shell. When tuning is achieved, strong coupling happens resulting in the optical cross-section spectra to exhibit a pair of split peaks. Upon adsorption of analytes on the surface of the core-shell nanostructure, the intensities of the two-peak change in opposite trends, which in principle allows core-shell nanostructures to be sensitive and quantitative ratiometric sensors. We proposed an optical experimental configuration to perform this measurement that is very likely to present a fast response time, much faster than what can be achieved with the traditional 
biosensors based on surface plasmon resonance of pure metal nanoparticles.

\section{Conflict of Interest}

The authors declare that they have no conflict of interest.

\section{Funding}

This work was supported by the Science and Technology Commission of Shanghai Municipality (I55207II500, I5ZRI4II700, I5ZRI4I0I00), the Joint Research Institute for Science and Society (JoRISS incubating project 2017-20I8), and the Program of Introducing Talents of Discipline to Universities (BI2024). BA and FA acknowledge support from Fondation pour la Recherche Médicale (DEI20I5I234404) and Agence National de la Recherche (ANR LightComb 2016-2018).

\section{Supplemental Material}

All supplemental material mentioned in the text, including five figures, is available in the online version of the journal.

\section{References}

I. S. Schlucker. "Surface-Enhanced Raman Spectroscopy: Concepts and Chemical Applications". Angew. Chem. Int. Ed. Engl. 2014. 53(19): 47564795.

2. J.N. Anker, W.P. Hall, O. Lyandres, N.C. Shah, J. Zhao, et al. "Biosensing with Plasmonic Nanosensors". Nat. Mater. 2008. 7(6): 442453.

3. M. Pelton. "Modified Spontaneous Emission in Nanophotonic Structures”. Nat. Photonics. 2015. 9(7): 427435.

4. J.J. Mock, D.R. Smith, S. Schultz. "Local Refractive Index Dependence of Plasmon Resonance Spectra from Individual Nanoparticles". Nano Lett. 2003. 3(4): 485 49I.

5. J. Becker, A. Trügler, A. Jakab, U. Hohenester, C. Sonnichsen. "The Optimal Aspect Ratio of Gold Nanorods for Plasmonic Bio-Sensing". Plasmonics. 2010. 5(2): 161167.

6. C.L. Nehl, H. Liao, J.H. Hafner. "Optical Properties of Star-Shaped Gold Nanoparticles”. Nano Lett. 2006. 6(4): 683688.

7. L.J. Sherry, S.H. Chang, G.C. Schatz, R.P. Van Duyne, B.J. Wiley, et al. "Localized Surface Plasmon Resonance Spectroscopy of Single Silver Nanocubes". Nano Lett. 2005. 5(I0): 20342038.

8. Y. Hu, R.C. Fleming, R.A. Drezek. "Optical Properties of Gold-SilicaGold Multilayer Nanoshells”. Opt. Express. 2008. 16(24): 1957919591.

9. J. Katyal, R.K. Soni. "Localized Surface Plasmon Resonance and Refractive Index Sensitivity of Metal-Dielectric-Metal Multilayered Nanostructures". Plasmonics. 2014. 9(5): II7I II8I.

I0. K.M. Mayer, J.H. Hafner. "Localized Surface Plasmon Resonance Sensors". Chem. Rev. 20II. III(6): 38283857.

II. C. Zhang, B.Q. Chen, Z.Y. Li, Y. Xia, Y.G. Chen. "Surface Plasmon Resonance in Bimetallic Core Shell Nanoparticles". J. Phys. Chem. C. 2015. II9(29): 1683616845.

I2. H. Shibata, K. Imakita, M. Fujii. "Fabrication of a Core Shell Shell Particle with a Quarter-Wave Thick Shell and its Optical Properties". RSC Adv. 2014. 4(6I): 3229332297.

13. F.X. Chen, X.C. Wang, D.L. Xia, L.S. Wang. "Design and Optimization of Ag-Dielectric Core-Shell Nanostructures for Silicon Solar Cells". AlP Adv. 2015. 5(9): 097I29.

14. T.J. Antosiewicz, S.P. Apell, T. Shegai. "Plasmon Exciton Interactions in a Core Shell Geometry: From Enhanced Absorption to Strong Coupling". ACS Photonics. 20I4. I(5): 454463.
15. T. Uwada, R. Toyota, H. Masuhara, T. Asahi. "Single Particle Spectroscopic Investigation on the Interaction between Exciton Transition of Cyanine Dye J-Aggregates and Localized Surface Plasmon Polarization of Gold Nanoparticles". J. Phys. Chem. C. 2007. III(4): I549 I552.

I6. G.P. Wiederrecht, G.A. Wurtz, J. Hranisavljevic. "Coherent Coupling of Molecular Excitons to Electronic Polarizations of Noble Metal Nanoparticles". Nano Lett. 2004. 4(II): 2I2I 2 I 25.

17. R. Bhandari. "Scattering Coefficients for a Multilayered Sphere: Analytic Expressions and Algorithms". Appl. Opt. 1985. 24(I3): 19601967.

18. J. Sinzig, M. Quinten. "Scattering and Absorption by Spherical Multilayer Particles". Appl. Phys. A: Mater. Sci. Process. 1994. 58(2): 157162.

19. B. Luk'yanchuk, N.I. Zheludev, S.A. Maier, N.J. Halas, P. Nordlander, et al. "The Fano Resonance in Plasmonic Nanostructures and Metamaterials". Nat. Mater. 2010. 9(9): 707 7I5.

20. B. Khlebtsov, N. Khlebtsov. "Ultrasharp Light-Scattering Resonances of Structured Nanospheres: Effects of Size-Dependent Dielectric Functions". J. Biomed. Opt. 2006. II(4): 215219.

2I. A.Q. Zhang, D.J. Qian, M. Chen. "Simulated Optical Properties of Noble Metallic Nanopolyhedra with Different Shapes and Structures". Eur. Phys. J. D. 2013. 67(II): 23I.

22. N.T. Fofang, T.H. Park, O. Neumann, N.A. Mirin, P. Nordlander, et al. "Plexcitonic Nanoparticles: Plasmon-Exciton Coupling in Nanoshell-JAggregate Complexes". Nano Lett. 2008. 8(10): 348I 3487.

23. N. Arnold, K. Piglmayer, A.V. Kildishev, T.A. Klar. "Spasers with Retardation and Gain Saturation: Electrodynamic Description of Fields and Optical Cross-Sections". Opt. Mater. Express. 2015. 5(II): 25462577

24. J.A. Gordon, R.W. Ziolkowski. "The Design and Simulated Performance of a Coated Nano-Particle Laser". Opt. Express. 2007. I5(5): 26222653.

25. K.M. McPeak, S.V. Jayanti, S.J.P. Kress, S. Meyer, S. lotti, et al. "Plasmonic Films Can Easily Be Better: Rules and Recipes". ACS Photonics. 2015. 2(3): 326333.

26. M. Rycenga, C.M. Cobley, J. Zeng, W. Li, C.H. Moran, et al. "Controlling the Synthesis and Assembly of Silver Nanostructures for Plasmonic Applications". Chem. Rev. 20II. III(6): 366937 I2.

27. K.A. Willets, R.P. Van Duyne. "Localized Surface Plasmon Resonance Spectroscopy and Sensing". Annu. Rev. Phys. Chem. 2007. 58: 267297.

28. S.H. Jeong, H. Choi, J.Y. Kim, T.W. Lee. "Silver-Based Nanoparticles for Surface Plasmon Resonance in Organic Optoelectronics". Part. Part. Syst. Charact. 2015. 32(2): 164175.

29. I.H. Malitson. "Interspecimen Comparison of the Refractive Index of Fused Silica". J. Opt. Soc. Am. 1965. 55(I0): 1205 I209.

30. S.A. Maier. "Localized Surface Plasmons". Plasmonics: Fundamentals and Applications. New York: Springer, 2007. Chap. 5, Pages 7377.

3I. C. Bonnand, J. Bellessa, J.C. Plenet. "Properties of Surface Plasmons Strongly Coupled to Excitons in an Organic Semiconductor Near a Metallic Surface”. Phys. Rev. B. 2006. 73(24): 245330.

32. J. Bellessa, C. Bonnand, J.C. Plenet, J. Mugnier. "Strong Coupling Between Surface Plasmons and Excitons in an Organic Semiconductor". Phys. Rev. Lett. 2004. 93(3): 036404.

33. G. Zengin, M. Wersäll, S. Nilsson, T.J. Antosiewicz, M. Kall, et al. "Realizing Strong Light-Matter Interactions between SingleNanoparticle Plasmons and Molecular Excitons at Ambient Conditions". Phys. Rev. Lett. 2015. II4(I5): I5740 I.

34. G. Zengin, G. Johansson, P. Johansson, T.J. Antosiewicz, M. Kall, et al. "Approaching the Strong Coupling Limit in Single Plasmonic Nanorods Interacting with J-Aggregates". Sci. Rep. 2013. 3: 3074.

35. S. Balci. "Ultrastrong Plasmon-Exciton Coupling in Metal Nanoprisms with J-Aggregates". Opt. Lett. 2013. 38(21): 4498450 I.

36. S. Balci, B. Kucukoz, O. Balci, A. Karatay, C. Kocabas, et al. "Tunable Plexcitonic Nanoparticles: A Model System for Studying Plasmon- 
Exciton Interaction from the Weak to the Ultrastrong Coupling Regime". ACS Photonics. 2016. 3(II): 20102016.

37. M. Wersall, J. Cuadra, T.J. Antosiewicz, S. Balci, T. Shegai. "Observation of Mode Splitting in Photoluminescence of Individual Plasmonic Nanoparticles Strongly Coupled to Molecular Excitons". Nano Lett. 2017. 17(I): 551558.

38. J. Bellessa, C. Symonds, J. Laverdant, J.M. Benoit, J.C. Plenet, et al. "Strong Coupling between Plasmons and Organic Semiconductors". Electronics. 20I4. 3(2): 303313.

39. S.J. Oldenburg, R.D. Averitt, S.L. Westcott, N.J. Halas. "Nanoengineering of optical resonances". Chem. Phys. Lett. 1998. 288(2 4): 243247.

40. P. Törmä, W.L. Barnes. "Strong Coupling Between Surface Plasmon Polaritons and Emitters: A Review". Rep. Prog. Phys. 20I5. 78(I): 01390I.

4I. E.M. Roller, C. Argyropoulos, A. Hogele, T. Liedl, M. Pilo-Pais. "Plasmon-Exciton Coupling Using DNA Templates". Nano Lett. 2016. 16(9): 59625966.
42. N. Zhou, M. Yuan, Y.H. Gao, D.S. Li, D. Yang. "Silver Nanoshell Plasmonically Controlled Emission of Semiconductor Quantum Dots in the Strong Coupling Regime". ACS Nano. 2016. 10(4): 4I54 4163.

43. Z. Chen, P. Zhan, Z.L. Wang, J.H. Zhang, W.Y. Zhang, et al. "Two- and Three-Dimensional Ordered Structures of Hollow Silver Spheres Prepared by Colloidal Crystal Templating”. Adv. Mater. 2004. 16(5): 417422.

44. A. Ben Moshe, G. Markovich. "Synthesis of Single Crystal Hollow Silver Nanoparticles in a Fast Reaction-Diffusion Process". Chem. Mater. 20II. 23(5): 12391245.

45. X.J. Zhao, R.P. Bagwe, W.H. Tan. "Development of Organic-DyeDoped Silica Nanoparticles in a Reverse Microemulsion". Adv. Mater. 2004. 16(2): 173 I76.

46. R.P. Bagwe, C.Y. Yang, L.R. Hilliard, W.H. Tan. "Optimization of DyeDoped Silica Nanoparticles Prepared Using a Reverse Microemulsion Method". Langmuir. 2004. 20(19): 83368342. 